\title{
Self-adaptation of Mutation Rates in Non-elitist Populations
}

\author{
Duc-Cuong Dang ${ }^{1}$, Per Kristian Lehre ${ }^{1}$ \\ School of Computer Science, University of Nottingham, United Kingdom \\ \{duc-cuong.dang, PerKristian.Lehre\}@nottingham.ac.uk
}

\begin{abstract}
The runtime of evolutionary algorithms (EAs) depends critically on their parameter settings, which are often problem-specific. Automated schemes for parameter tuning have been developed to alleviate the high costs of manual parameter tuning. Experimental results indicate that self-adaptation, where parameter settings are encoded in the genomes of individuals, can be effective in continuous optimisation. However, results in discrete optimisation have been less conclusive. Furthermore, a rigorous runtime analysis that explains how self-adaptation can lead to asymptotic speedups has been missing. This paper provides the first such analysis for discrete, population-based EAs. We apply level-based analysis to show how a self-adaptive EA is capable of fine-tuning its mutation rate, leading to exponential speedups over EAs using fixed mutation rates.
\end{abstract}

\section{Introduction}

An obstacle when applying Evolutionary Algorithms (EAs) is that their efficiency depends crucially, and sometimes unpredictably, on their parameter settings, such as population size, and mutation rates. Parameter tuning [7], where the parameters are fixed before running the algorithm, is the most common way of choosing the parameters. A weakness with parameter tuning is that optimal parameter settings may depend on the current state of the search process. In contrast, parameter control allows the parameters to change during the execution of the algorithm, e.g. according to a fixed schedule (e.g. as in simulated annealing), through feedback from the search, or via self-adaptation [7]. Adaptive parameters can be essential and advantageous (e.g. covariance-matrix adaptation [8]) in continuous search spaces. In discrete spaces, it has been shown that changing the mutation rate as a function of the current fitness [2] can improve the runtime, and the $1 / 5$-rule has been used to adapt the population size [5].

While previous studies have shown the benefit of adaptive parameters, only global parameters were analysed. Our focus is different, we look at the so-called "evolution of evolution" or true self-adaptation [7], in which the parameter is encoded in the genome of individual solutions. As far as we know, the existing studies on this topic from the EC literature is mostly experimental $[1,7,12]$, or about proving the convergence of the population model at their limit [1], i. e. infinite population. 
We study how mutation rates can evolve within a non-elitist population, where the mutation rate of each individual is encoded by its own genome. The rate at which the mutation rate mutates is specified by a strategy parameter $p$. In endogenous control, the strategy parameter is itself evolved $[1,13]$. Here, we consider exogenous control of the strategy parameter $p$, where the value of the parameter is fixed before the run. Our contribution is twofold: using LEADINGONES as a benchmark, we provide the necessary and sufficient conditions, especially those on $p$, for self-adaptation to work; by making a small modification of the function, we show that self-adaptation is essential in optimising the modified function, more precisely that a single mutation rate or uniform mixing of mutation rates requires exponential time, while self-adaptation is efficient. We also prove that a non-elitist EA can outperform the $(\mu+\lambda)$ EA.

\section{Preliminaries}

For any $n \in \mathbb{N}$, define $[n]:=\{1, \ldots, n\}$. The natural logarithm is denoted by $\ln (\cdot)$, and the logarithm to the base 2 is denoted by $\log (\cdot)$. For $x \in\{0,1\}^{n}$, we write $x(i)$ for the $i$-th bit value. The Hamming distance is denoted by $\mathrm{H}(\cdot, \cdot)$ and the Iverson bracket by [.]. Given a partition of a search space $\mathcal{X}$ into $m$ ordered "levels" $\left(A_{1}, \ldots, A_{m}\right)$, we define $A_{\geq j}:=\cup_{i=j}^{m} A_{i}$. A population is a vector $P \in \mathcal{X}^{\lambda}$, where the $i$-th element $P(i)$ is called the $i$-th individual. Given $A \subseteq \mathcal{X}$, we let $|P \cap A|:=|\{i \mid P(i) \in A\}|$ be the number of individuals in population $P$ that belong to the subset $A$. All algorithms considered here are of the form of Algorithm 1 [4]. A new population $P_{t+1}$ is generated by independently sampling $\lambda$ individuals from an existing population $P_{t}$ according to $p_{\text {sel }}$, and perturbing each of the sampled individuals by a variation operator $p_{\text {mut }}$. The selection mechanism $p_{\text {sel }}$ implicitly embeds a fitness function $g: \mathcal{Y} \rightarrow \mathbb{R}$.

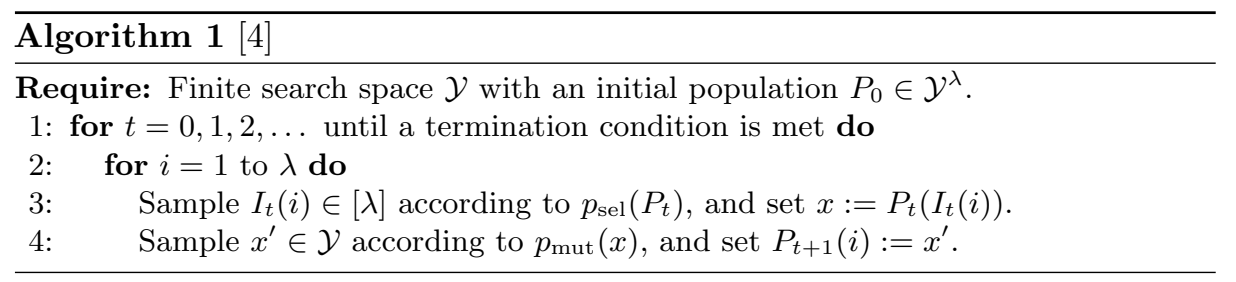

We consider the standard bitwise mutation operator, where for any pair of bitstrings $x, x^{\prime} \in\{0,1\}^{n}$ and any mutation rate $\chi \in(0, n]$, the probability of obtaining $x^{\prime}$ from $x$ is $\operatorname{Pr}\left(x^{\prime}=\operatorname{mut}(x, \chi)\right)=(\chi / n)^{H\left(x, x^{\prime}\right)}(1-\chi / n)^{n-H\left(x, x^{\prime}\right)}$. To model the parameter control problem, we assume that Algorithm 1 must choose mutation rates from a predefined set $\mathcal{M}$.

Uniform mixing, denoted $p_{\text {mut }}^{\text {mix }}$, chooses the mutation rate $\chi$ uniformly at random from the set $\mathcal{M}$ every time an individual is mutated, $p_{\text {mut }}^{\text {mix }}(x):=$ $\operatorname{mut}(x, \chi)$, where $\chi \sim \operatorname{Unif}(\mathcal{M})$.

The special case of $|\mathcal{M}|=1$, i.e. a fixed mutation rate, has been studied extensively $[4,11]$. 
Here, we focus on $|\mathcal{M}|>1$. It is known that such mixing of mutation operators can be beneficial $[10,6]$.

Self-adaptation uses an extended search space $\mathcal{Y}=\mathcal{X} \times \mathcal{M}$, where each element $(x, \chi)$ consists both of a search point $x \in \mathcal{X}$ and a mutation rate $\chi \in \mathcal{M}$. A fitness function $g: \mathcal{Y} \rightarrow \mathbb{R}$ is defined by $g((x, \chi)):=f(x)$ for all $(x, \chi) \in \mathcal{Y}$. The mutation operator $p_{\text {mut }}$ is written as $p_{\text {mut }}^{\text {adapt }}$ and it is parameterised by a globally fixed parameter $p \in(0,1 / 2]$ such that $p_{\text {mut }}^{\text {adapt }}((x, \chi)):=\left(x^{\prime}, \chi^{\prime}\right)$ where $\chi^{\prime}=\chi$ with probability $1-p$, and $\chi^{\prime} \sim \operatorname{Unif}(\mathcal{M} \backslash\{\chi\})$ otherwise, and $x^{\prime}=\operatorname{mut}\left(x, \chi^{\prime}\right)$.

We analyse the runtime of Algorithm 1 using the level-based theorem [3]. This theorem applies to any population-based process where the individuals in $P_{t+1}$ are sampled independently from the same distribution $D\left(P_{t}\right)$, where $D$ maps populations $P_{t}$ to distributions over the search space $\mathcal{X}$. In Algorithm 1, the map is $D=p_{\text {mut }} \circ p_{\text {sel }}$, i.e., composition of selection and mutation.

Theorem 1 ([3]). Given a partition $\left(A_{1}, \ldots, A_{m+1}\right)$ of $\mathcal{X}$, define $T:=\min \{t \lambda \mid$ $\left.\left|P_{t} \cap A_{m+1}\right|>0\right\}$ to be the first point in time that elements of $A_{m+1}$ appear in $P_{t}$ of Algorithm 1. If there exist parameters $z_{1}, \ldots, z_{m}, z_{*} \in(0,1], \delta>0$, a constant $\gamma_{0} \in(0,1)$ and a function $z_{0}:\left(0, \gamma_{0}\right) \rightarrow \mathbb{R}$ such that for all $j \in[m], P \in \mathcal{X}^{\lambda}$, $y \sim D(P)$ and $\gamma \in\left(0, \gamma_{0}\right]$ we have

(G1) $\operatorname{Pr}\left(y \in A_{\geq j}|| P \cap A_{\geq j-1} \mid \geq \gamma_{0} \lambda\right) \geq z_{j} \geq z_{*}$

(G2) $\operatorname{Pr}\left(y \in A_{\geq j}|| P \cap A_{\geq j-1}\left|\geq \gamma_{0} \lambda,\right| P \cap A_{\geq j} \mid \geq \gamma \lambda\right) \geq z_{0}(\gamma) \geq(1+\delta) \gamma$

(G3) $\lambda \geq \frac{2}{a} \ln \left(\frac{16 m}{a c \varepsilon z_{*}}\right)$ with $a=\frac{\delta^{2} \gamma_{0}}{2(1+\delta)}, \varepsilon=\min \{\delta / 2,1 / 2\}$ and $c=\varepsilon^{4} / 24$

then $\mathbf{E}[T] \leq(2 / c \varepsilon)\left(m \lambda(1+\ln (1+c \lambda))+\sum_{j=1}^{m} 1 / z_{j}\right)$.

We apply the negative drift theorem for populations [9] to obtain tail bounds on the runtime of Algorithm 1. For any individual $P_{t}(i)$ in Algorithm 4 where $t \in \mathbb{N}$ and $i \in[\lambda]$, define $R_{t}(i):=\left|\left\{j \in[\lambda] \mid I_{t}(j)=i\right\}\right|$, i.e., the number of times the individual was selected. We define the reproductive rate of the individual $P_{t}(i)$ to be $\mathbf{E}\left[R_{t}(i) \mid P_{t}\right]$, i.e., the expected number of offspring from individual $P_{t}(i)$. Informally, the theorem states that if all individuals close to a given search point $x^{*} \in \mathcal{X}$ have reproductive rate below a certain threshold $\alpha_{0}$, then the algorithm needs exponential time to reach $x^{*}$. The threshold depends on the mutation rate. Here, we derive a variant of this theorem for algorithms that use multiple mutation rates. In particular, we assume that the algorithm uses $m$ mutation rates, where mutation rate $\chi_{i} / n$ for $i \in[m]$ is chosen with probability $q_{i}$. The proof of this theorem is similar to that of Theorem 4 in [9], and thus omitted

Theorem 2. For any $x^{*} \in\{0,1\}^{n}$, define $T:=\min \left\{t \mid x^{*} \in P_{t}\right\}$, where $P_{t}$ is the population of Algorithm 1 at time $t \in \mathbb{N}$. If there exist constants $\alpha_{0}, c, c^{\prime}, \delta>0$ such that with probability $1-e^{-\Omega(n)}$

- the initial population satisfies $H\left(P_{0}, x^{*}\right) \geq c^{\prime} n$

- for all $t \leq e^{c n}$ and $i \in[\lambda]$, if $H\left(P_{t}(i), x^{*}\right) \leq c^{\prime} n$, then the reproductive rate of individual $P_{t}(i)$ is no more than $\alpha_{0}$, 
$-\sum_{j=1}^{m} q_{j} e^{-\chi_{j}} \leq(1-\delta) / \alpha_{0}$, and $\max _{j} \chi_{j} \leq \chi_{\max }$ for a constant $\chi_{\max }$,

then $\operatorname{Pr}\left(T \leq e^{c^{\prime \prime} n}\right)=e^{-\Omega(n)}$ for a constant $c^{\prime \prime}>0$.

Proof (of Theorem 2). We apply Theorem 1 in [9]. The first condition holds immediately. We use the distance function $g(x):=H\left(x, x^{*}\right)$ over the interval $[b(n) / 2, b(n)]$, where $b(n):=n /\left(e^{2 \kappa}-1\right)$ and $\kappa:=\max \left\{\ln (2), \ln \left(\frac{4 \chi_{\max }}{\ln (1+\delta)}\right)\right\}$. Without loss of generality, we assume that $x^{*}=1^{n}$, hence $g(x)$ is the number of 0-bits in $x$.

For the second condition, the drift of the process $\Delta(i):=\left(g\left(X_{t+1}\right)-g\left(X_{t}\right) \mid\right.$ $\left.g\left(X_{t}\right)=i\right)$ is a sum $\Delta(i)=\Delta^{+}(i)-\Delta^{-}(i)$ of two binomially distributed random variables, the number of 1-bits flipped $\Delta^{+}(i) \sim \operatorname{Bin}(n-i, Q)$, and the number of 0 -bits flipped, where $Q \sim q$. For $i<b(n)$, we use $\exp \left(n p\left(e^{\kappa}-1\right)\right)$ as an upper bound on the mgf of a binomially distributed random variable with parameters $n$ and $p$, and get

$$
\begin{aligned}
\mathbf{E}\left[e^{-\kappa \Delta(i)} \mid X_{t}\right] & =\mathbf{E}\left[\mathbf{E}\left[e^{-\kappa\left(\Delta^{-}(i)-\Delta^{+}(i)\right)} \mid Q\right] \mid X_{t}\right] \\
& \leq \sum_{j=1}^{m} q_{j} \exp \left((n-i)\left(\chi_{j} / n\right)\left(e^{-\kappa}-1\right)+i\left(\chi_{j} / n\right)\left(e^{\kappa}-1\right)\right) \\
& \left.=\sum_{j=1}^{m} q_{j} \exp \left(-\chi_{j}\left(1-e^{-\kappa}\right)+(i / n) \chi_{j}\left(e^{\kappa}-e^{-\kappa}\right)\right)\right) \\
& =\sum_{j=1}^{m} q_{j} \exp \left(-\chi_{j}\left(1-2 e^{-\kappa}\right)\right) .
\end{aligned}
$$

Noting that $e^{-\kappa} \leq \ln (1+\delta) /\left(4 \chi_{\max }\right)$, we get

$$
\begin{aligned}
\mathbf{E}\left[e^{-\kappa \Delta(i)} \mid X_{t}\right] & \left.\leq \sum_{j=1}^{m} q_{j} \exp \left(-\chi_{j}\right) \exp \left(\chi_{\max } 2 e^{-\kappa}\right)\right) \\
& \leq \sum_{j=1}^{m} q_{j} \exp \left(-\chi_{j}\right)(1+\delta)^{1 / 2} \leq \frac{1}{\alpha_{0}(1+\delta)^{1 / 2}} .
\end{aligned}
$$

The second condition is then satisfied. The third and fourth conditions can be satisfied for any mutation rate $\chi / n$ for appropriate positive constants $\delta_{2}, \delta_{3} \in(0,1)$ and $D(n)$, as long as $\kappa(n) \geq \ln (2)$ (see the proof of Theorem 4 in [9]).

\section{General negative results}

Using Theorem 2, we can now show general negative results for uniform mixing and self-adaptation of two mutation rates for any function with a unique global optimum $x^{*}$, assuming that the initial population is positioned sufficiently far away from $x^{*}$. The following theorem is a special case of Theorem 2 for $|\mathcal{M}|=1$. 
Theorem 3. The runtime of Algorithm 1 with reproductive rate $\alpha_{0}$ and mutation rate $\chi_{\text {high }} / n \geq\left(\ln \left(\alpha_{0}\right)+\delta\right) / n$ for some constant $\delta>0$ satisfies $\operatorname{Pr}\left(T \leq e^{c n}\right)=$ $e^{-\Omega(n)}$ on any function with a unique global optimum $x^{*}$ assuming that $\mathrm{H}\left(P_{0}, x^{*}\right) \geq$ $c^{\prime} n$ for two constants $c>0$ and $c^{\prime} \in(0,1)$.

For binary tournament and $(\mu, \lambda)$-selection, $\alpha_{0}$ is bounded from above by 2 and $\lambda / \mu$ respectively. Hence, any mutation rate above $\ln (2)$ for 2 -tournament selection and $\ln (\lambda / \mu)$ for $(\mu, \lambda)$-selection by a constant renders the EA inefficient.

For $|\mathcal{M}|=2$, we have the following general result, again due to Theorem 2 .

Theorem 4. Consider Algorithm 1 with reproductive rate $\alpha_{0}$ and mutation rates $\chi_{\text {low }} / n$ and $\chi_{\text {high }} / n$. If there exist constants $\delta_{1}, \delta_{2}, \varepsilon>0$ such that

- $\chi_{\text {low }} \geq \ln \left(\alpha_{0}\right)-\ln \left(1+\delta_{1}\right)$ and $\chi_{\text {high }} \geq \ln \left(\alpha_{0}\right)-\ln \left(1-\delta_{2}\right)$,

- the EA chooses mutation rate $\chi_{\text {high }}$ with probability at least $\frac{\delta_{1}(1+\varepsilon)}{\delta_{1}+\delta_{2}}$,

then $\operatorname{Pr}\left(T \leq e^{c n}\right)=e^{-\Omega(n)}$ on any function with a unique optimum $x^{*}$ given that $\mathrm{H}\left(P_{0}, x^{*}\right) \geq c^{\prime} n$ for some constants $c^{\prime}, c>0$

Proof (of Theorem 4). We have

$$
\begin{aligned}
q_{1} e^{-\chi_{\text {low }}}+q_{2} e^{-\chi_{\mathrm{high}}} & =\left(1-q_{2}\right) e^{-\chi_{\mathrm{low}}}+q_{2} e^{-\chi_{\mathrm{high}}} \\
& \leq\left(\left(1-q_{2}\right)\left(1+\delta_{1}\right)+q_{2}\left(1-\delta_{2}\right)\right) / \alpha_{0} \\
& =\left(1+\delta_{1}-q_{2}\left(\delta_{1}+\delta_{2}\right)\right) / \alpha_{0} \leq\left(1-\varepsilon \delta_{1}\right) / \alpha_{0},
\end{aligned}
$$

which by Theorem 2 implies the result.

Uniform mixing selects the mutation rate $\chi_{\text {high }} / n$ with probability $1 / 2$. Thus, if $\delta_{1} /\left(\delta_{1}+\delta_{2}\right)$ is below $1 / 2$ by a constant then the EA is inefficient. For example, in binary tournament, the setting $\chi_{\text {low }} \geq \ln (3 / 2)-\ln (100 / 99)$ and $\chi_{\text {high }} \geq \ln 3+$ $\ln (33 / 32)$ satisfies the conditions of the theorem for $\delta_{1}=103 / 297, \delta_{2}=105 / 297$ and $\delta_{1} /\left(\delta_{1}+\delta_{2}\right)=103 / 208<1 / 2$. Theorem 8 later on will show the efficiency of self-adaptation in this setting in contrast to the uniform mixing. In self-adaptation, $\chi_{\text {high }} / n$ is selected with at least probability $p$, thus self-adaptation does not work if $p$ is above $\delta_{1} /\left(\delta_{1}+\delta_{2}\right)$ by a constant.

\section{Robust self-adaptation}

The previous section showed how critically non-elitist EAs depend on having appropriate mutation rates. A slightly too high mutation rate $\chi_{\text {high }}$ can lead to an exponential increase in runtime. Uniform mixing of mutation rates can fail if the of mutation rates $\mathcal{M}$ contains one such high mutation rate, even though the set also contains an appropriate mutation rate $\chi_{\text {low }}$.

Self-adaptation has a similar problem if the strategy parameter $p$ is chosen too high. However, we will prove for a simple, unimodal fitness function that for a sufficiently small strategy parameter $p$, self-adaptation becomes highly robust, and is capable of fine-tuning the mutation rate. For the rest of this section, 
we consider a set of two mutation rates $\mathcal{M}=\left\{\chi_{\text {low }}, \chi_{\text {high }}\right\}$ which for arbitrary parameters $\ell \in[n]$ and $\varepsilon>0$ are defined by $\left(1-\frac{\chi_{\text {high }}}{n}\right)^{\ell}<\frac{\mu}{\lambda} \leq\left(1-\frac{\chi_{\text {high }}}{n}\right)^{\ell-1}$ and $\frac{\mu}{\lambda}(1+\varepsilon) \leq\left(1-\frac{\chi_{\text {low }}}{n}\right)^{n}$. By the previous section, if $\ell$ is chosen sufficiently small, and hence $\chi_{\mathrm{high}}$ sufficiently high, then uniform mixing will fail on any problem with a unique optimum. In contrast, using a Chernoff and a union bound, the following lemma shows that individuals that have chosen $\chi_{\text {high }}$ will quickly vanish from a self-adapting population, and the population will be dominated by individuals choosing the appropriate mutation parameter $\chi_{\text {low }}$.

Lemma 1. Let $Y_{t}:=\left|P_{t} \cap A_{-1}\right|$ where $P_{t}$ is the population of Algorithm 1 at time $t \in \mathbb{N}$ with $(\mu, \lambda)$-selection on LEADINGONES and the set $A_{-1}$ is as defined in Eq. (1). Then $\operatorname{Pr}\left(Y_{t} \geq \max \left((3 / 4) \mu,(1-p / 3)^{t} Y_{0}\right)\right) \leq t \cdot e^{-\Omega(\lambda)}$ for all $t \in \mathbb{N}$.

Proof (of Lemma 1). For an upper bound, we assume that search points in $B$ have higher fitness than search points outside $B$. The probability of producing a $B$-individual with $(\mu, \lambda)$-selection is at most

$$
\begin{aligned}
\left(1-\frac{\chi_{\mathrm{high}}}{n}\right)^{\ell}\left(\frac{Y_{t}}{\mu}(1-p)+\left(1-\frac{Y_{t}}{\mu}\right) p\right) & \leq\left(\frac{\mu}{\lambda}\right)\left(\frac{Y_{t}}{\mu}(1-2 p)+p\right) \\
& \leq\left(\frac{\mu}{\lambda}\right)\left(\max \left(\frac{3}{4}, \frac{Y_{t}}{\mu}\right)(1-2 p)+p\right) \\
& \leq \max \left(\frac{3 \mu}{4 \lambda}, \frac{Y_{t}}{\lambda}\right)(1-2 p / 3)=: p_{s}
\end{aligned}
$$

Hence, $Y_{t+1}$ is stochastically dominated by a random variable $Z \sim \operatorname{Bin}\left(\lambda, p_{s}\right)$. It now follows by a Chernoff bound that

$$
\begin{aligned}
\operatorname{Pr}\left(Y_{t+1} \geq \max \left(3 \mu / 4,(1-p / 3) Y_{t}\right)\right) & \leq \operatorname{Pr}\left(Z \geq \mathbf{E}[Z]\left(1+\frac{p}{3-2 p}\right)\right) \\
& \leq \exp \left(-\frac{p^{2} \mu}{12(3-2 p)}\right) .
\end{aligned}
$$

The proof now follows by induction on $t$ and a union bound.

Theorem 5. Algorithm 1 with $(\mu, \lambda)$-selection where $\lambda \geq c \ln (n)$ for a sufficiently large constant $c>0$, and self-adaptation from the set $\mathcal{M}=\left\{\chi_{\text {low }}, \chi_{\text {high }}\right\}$ using a sufficiently small constant strategy parameter $p$ satisfying $(1+\varepsilon)(1-p) \geq 1+p \varepsilon$ has expected runtime $O\left(n \lambda \log (\lambda)+n^{2}\right)$ on LEADINGONES.

Proof. We partition the search space into the following $n+2$ levels

$$
A_{j}:= \begin{cases}\left\{\left(x, \chi_{\text {high }}\right) \mid \operatorname{Lo}(y) \geq \ell\right\} & \text { if } j=-1 \\ \left\{\left(x, \chi_{\text {low }}\right),\left(x, \chi_{\text {high }}\right) \mid \operatorname{Lo}(x)=j\right\} & \text { if } 0 \leq j \leq \ell-1 \\ \left\{\left(x, \chi_{\text {low }}\right) \mid \operatorname{LO}(x)=j\right\} & \text { if } \ell \leq j \leq n .\end{cases}
$$

The special level $A_{-1}$ contains search points with too high mutation rate. We first estimate the expected runtime assuming that there are never more than 
(3/4) $\mu$ individuals in level $A_{-1}$. In the end, we will account for the generations where this assumption does not hold.

We now show that conditions (G1) and (G2) of the level-based theorem hold for the parameters $\gamma_{0}:=(1 / 8)(\mu / \lambda), \delta:=p \varepsilon$, and $z_{j}=\Omega(1 / n)$. Assume that the current population has at least $\gamma_{0} \lambda=\mu / 8$ individuals in $A_{\geq j-1}$ and $\gamma \lambda<\gamma_{0} \lambda$ individuals in $A_{\geq j}$, for $0 \leq j \leq n$ and $\gamma \in\left[0, \gamma_{0}\right)$. If $0 \leq j \leq \ell-1$, then an individual can be produced in levels $A_{\geq j}$ if one of the $\gamma \lambda$ individuals in these levels is selected, and none of the first $j$ bits are mutated. Assuming in the worst case that the selected individual has chosen the high mutation rate, the probability of this event is at least $\left(\frac{\gamma \lambda}{\mu}\right)\left(\left(1-\frac{\chi_{\text {high }}}{n}\right)^{j}(1-p)+\left(1-\frac{\chi_{\text {low }}}{n}\right)^{j} p\right)$ $>\left(\frac{\gamma \lambda}{\mu}\right)\left(\left(1-\frac{\chi_{\mathrm{high}}}{n}\right)^{\ell-1}(1-p)+\left(1-\frac{\chi_{\text {low }}}{n}\right)^{n} p\right) \geq \gamma(1+p \varepsilon)$. All individuals in levels $j \geq \ell$ use the low mutation rate. Hence, an individual in levels $A_{\geq j}$ can be produced by selecting one the $\gamma \lambda$ individuals in this level, not change the mutation rate, and not flip any of the first $j \leq n$ leading 1-bits. The probability of this event is at least $\frac{\gamma \lambda}{\mu}\left(1-\frac{\chi_{\text {low }}}{n}\right)^{j}(1-p)>\frac{\gamma \lambda}{\mu}\left(\frac{\mu}{\lambda}(1+\varepsilon)(1-p)\right) \geq \gamma(1+\delta)$. Condition (G2) is therefore satisfied for all levels. For condition (G1), assume that the population does not contain any individuals in $A_{\geq j}$. Then in the worst case, it suffices to select one of the at least $\gamma_{0} \lambda$ individuals in level $A_{j}$, switch the mutation rate, and only flip the first 0 -bit and no other bits. The probability of this event is higher than $\frac{\gamma_{0} \lambda}{\mu}\left(\frac{\chi_{\text {low }}}{n}\right)\left(1-\frac{\chi_{\text {high }}}{n}\right)^{n-1} p=\Omega(1 / n)$.

Condition (G3) holds for any population size $\lambda \geq c \ln (n)$ and a sufficiently large constant $c$, because $\gamma_{0}$ and $\delta$ are constants. It follows that the expected number of generations until the optimum is found is $t_{1}(n)=O\left(n \log (\lambda)+n^{2} / \lambda\right)$. By Markov's inequality, the probability that the algorithm has not found the optimum after $2 t_{1}(n)$ generations is less than $1 / 2$.

Finally, we account for the generations with more than $(3 / 4) \mu$ individuals in level $A_{-1}$. We call a phase good if after $t_{0}(n)=O(\log (\lambda))$ generations and for the next $2 t_{1}(n)$ generations, there are fewer than $(3 / 4) \mu$ individuals in level $A_{-1}$. By Lemma 1 , a phase is good with probability $1-\left(t_{0}(n)+2 t_{1}(n)\right) \cdot e^{-\Omega(\lambda)}=\Omega(1)$, for $\lambda \geq c \ln (n)$ and $c$ a sufficiently large constant. By the level-based analysis, the optimum is found with probability at least $1 / 2$ during a good phase. Hence, the expected number of phases required to find the optimum is $O(1)$. The theorem now follows by keeping in mind that each generation costs $\lambda$ evaluations.

We have shown that the EA can self-adapt to choose the low mutation parameter $\chi_{\text {low }}$ when required. Nevertheless, uniform mixing of mutation rates with a sufficiently small $\chi_{\text {low }}$ could achieve the same asymptotic performance. Furthermore, naively picking a mutation rate from the beginning also has a constant probability of optimising the function in polynomial time. Our aim is therefore to show that there exists a setting for which all the above approaches, except self-adaptation, fail. To prove this, we have identified a problem $f_{m}$ where a high mutation rate is required in one part of the search space, and a low mutation rate is required in another part. For $1 \leq m<n$, define $f_{m}\left(0^{n}\right):=m$ and $f_{m}(x):=\operatorname{LEADINGONES}(x)$ for all $x \neq 0^{n}$. We call the local optimum $0^{n}$ the peak, and assume that all individuals in the initial population are peak individuals. 
It is clear that the elitist algorithm $(\mu+\lambda)$ EA without any diversity mechanism will only accept a search point if it has at least $m$ leading 1-bits.

Theorem 6. Starting at $0^{n}$, the $(\mu+\lambda)$ EA has expected runtime $n^{\Omega(m)}$ on $f_{m}$.

To reach the optimal search point more efficiently, it is necessary to accept worse individuals into the population, e.g. a non-elitist selection scheme should be investigated. Since $f_{m}$ has a unique global optimum, either using only a too high mutation rate or uniformly mixing a correct mutation rate with a too high one can lead to exponential runtime as discussed above. Analogously to the $(\mu+\lambda) \mathrm{EA}$, we also prove that using a too low mutation rate fails because the population is trapped on the peak (e.g. due to Theorem 2, individuals fell off the peak have too low reproductive rate to optimise $m$ leading 1-bits). Subsequent proofs use the two functions $q(i):=\left(1-\chi_{\text {low }} / n\right)^{i}$ and $r(i):=\left(1-\chi_{\text {high }} / n\right)^{i}$, which are the probabilities of not flipping the first $i \in[n]$ bits using mutation rate $\chi_{\text {low }} / n$ and $\chi_{\text {high }} / n$ respectively. Clearly, $q(i)$ and $r(i)$ are monotonically decreasing in $i$. We also use the function $\beta(\gamma):=2 \gamma(1-\gamma / 2)$, which is the probability that binary tournament selection chooses one of the $\gamma \lambda$ fittest individuals.

Theorem 7. The runtime of Algorithm 1 on $f_{m}$ with tournament size 2, initialised with the population at $0^{n}$ and with fixed mutation rate $\chi \leq \ln (3 / 2)-\varepsilon$ for any constant $\varepsilon \in(0, \ln (3 / 2))$ satisfies $\operatorname{Pr}\left(T \leq e^{c n}\right)=e^{-\Omega(\lambda)}$ for a constant $c>0$.

Proof (of Theorem 7). We will prove that with probability $1-e^{-\Omega(\lambda)}$, all individuals during the first $e^{c n}$ generations have less than $m$ leading 1-bits, where $c>0$ is a constant. Clearly, this stronger statement implies the theorem.

Choose the parameter $\delta \in(0,1)$ such that $\ln ((1+\delta) /(1-\delta))=\varepsilon$. We first show by induction that with probability $1-e^{-\Omega(\lambda)}$, there are at least $(\lambda / 2)(1+\delta / 2)$ peak-individuals in each of the first $e^{c \lambda}$ generations, and we call the run of the algorithm a failure otherwise. By Lemma 3, the probability of not mutating any bits when $n \in \mathbb{N}$ is sufficiently large is $q(n) \geq e^{-\chi}(1-\delta) \geq(2 / 3)(1+\delta)$. Assume that there are $\gamma \lambda \geq \lambda / 2$ peak individuals in the current population. A peak individual is produced if a peak individual is selected and none of its bits are flipped. The probability of this event is at least $\beta(\gamma) q(n) \geq \beta(1 / 2) q(n)=$ $(1 / 2)(2-1 / 2)(2 / 3)(1+\delta)=(1 / 2)(1+\delta)$, where the first inequality uses the fact that $\beta(\gamma)$ is strictly increasing in $\gamma$ over the interval $[0,1]$. Hence, by a Chernoff bound, the probability that the next generation contains less than $(\lambda / 2)(1+\delta / 2)$ peak individuals is $e^{-\Omega(\lambda)}$. By induction and a union bound, the bound $e^{-\Omega(\lambda)}$ also holds for the next $e^{c \lambda}$ generations, if $c>0$ is a sufficiently small constant.

We now assume that the run is not a failure. Furthermore, we assume that the algorithm is optimising the function $g(x):=\min \left(m, f_{m}(x)\right)$ instead of $f_{m}$. Clearly, the time to reach at least $m$ leading 1-bits is the same, whether the algorithm optimises $g$ or $f_{m}$. Assuming that there are more than $(\lambda / 2)(1+\delta / 2)$ peak individuals, the reproductive rate of any non-peak individual is always less than $\lambda\left(2(1 / \lambda)(1-1 / \lambda-(1 / 2)(1+\delta / 2))+(1 / \lambda)^{2}\right)<1-\delta / 2=: \alpha_{0}$. 
For non-peak individuals, the last $n-m$ bit-positions are irrelevant when the algorithm optimises $g$. We can therefore apply the negative drift theorem (Theorem 2) with respect to the algorithm limited to the first $m$ bit positions only. The variation operator in this algorithm flips each of the $m$ bits independently with probability $\chi^{\prime} / m$, where $\chi^{\prime}=\chi_{\text {low }}(m / n)$. Hence, we have $e^{-\chi^{\prime}}<1=(1-\delta / 2) / \alpha_{0}$, and the conditions of the theorem are satisfied.

Theorem 8. If $\mathcal{M}=\left\{\chi_{\text {low }}, \chi_{\text {high }}\right\}$ where $\chi_{\text {low }}:=\ln (3 / 2)-\varepsilon$ for any constant $\varepsilon \in(0, \ln (100 / 99))$, and $\ln (3) \leq \chi_{\text {high }}=O(1)$, then there exists an $m \in \Theta(n)$ such that Algorithm 1 starting with the population at $0^{n}$, with tournament size 2 , population size $\lambda \geq c \ln n$ for some constant $c>0$ and self-adaptation of $\mathcal{M}$ with $p=1 / 20$ has expected runtime $O\left(n \lambda \log (\lambda)+n^{2}\right)$ on $f_{m}$.

Our intuition is that with sufficiently high mutation rate, some individuals fall off the peak and form a sub-population which optimises the LEADINGONES part of the problem. This will happen if the selective pressure is not too high. However, at the same time, the population should be able to reach the optimal search point $1^{n}$ after escaping the local optimum. Here we used the level-based technique to infer constraints on the mutation rates and the strategy parameter $p$. The proof idea follows closely from these observations.

We will need the following result to limit the number of individuals at unfavourable portions of the search space, i.e. too many individuals in those portions will prevent the algorithm from moving in the right direction.

Lemma 2. Given any subset $A \subset \mathcal{X}$, let $Y_{t}:=\left|P_{t} \cap A\right|$ be the number of individuals in generation $t \in \mathbb{N}$ of Algorithm 1 with tournament size 2, that belong to subset $A$. If there exist three parameters $\rho, \sigma, \varepsilon \in(0,1)$ such that $\operatorname{Pr}\left(p_{\text {mut }}(y) \in A\right) \leq \rho$ for all $y \in A$ and $\operatorname{Pr}\left(p_{\text {mut }}(y) \in A\right) \leq \sigma \gamma_{*}-\varepsilon$ for all $y \notin A$, where $\gamma_{*}:=2-(1-\sigma) / \rho$, then $\operatorname{Pr}\left(Y_{t} \geq \max \left(\gamma_{*} \lambda,(1-\varepsilon / 2)^{t} Y_{0}\right)\right) \leq t \cdot e^{-\Omega(\lambda)}$.

Proof (of Lemma 2). For an upper bound, we assume that all search points in $A$ have higher fitness than search points in $\mathcal{X} \backslash A$. The probability of selecting an individual in $A$ is therefore $\beta\left(Y_{t} / \lambda\right)$. The probability that any given offspring in generation $t+1 \leq e^{c \lambda}-1$ belongs to subset $A$ is no more than

$$
\begin{aligned}
\beta\left(Y_{t} / \lambda\right) \rho+\sigma \gamma_{*}-\varepsilon & \leq \beta\left(\max \left(\gamma_{*}, Y_{t} / \lambda\right)\right) \rho+\sigma \gamma_{*}-\varepsilon \\
& \leq 2 \max \left(\gamma_{*}, Y_{t} / \lambda\right)\left(1-\max \left(\gamma_{*}, Y_{t} / \lambda\right) / 2\right) \rho+\sigma \gamma_{*}-\varepsilon \\
& \leq 2 \max \left(\gamma_{*}, Y_{t} / \lambda\right)\left(1-\gamma_{*} / 2\right) \rho+\sigma \gamma_{*}-\varepsilon \\
& =\max \left(\gamma_{*}, Y_{t} / \lambda\right)(1-\sigma)+\sigma \gamma_{*}-\varepsilon \\
& \leq \max \left(\gamma_{*}, Y_{t} / \lambda\right)(1-\varepsilon)=: p_{s} .
\end{aligned}
$$


Hence, $Y_{t+1}$ is stochastically dominated by the random variable $Z \sim \operatorname{Bin}\left(\lambda, p_{s}\right)$. It now follows by a Chernoff bound that

$$
\begin{aligned}
\operatorname{Pr}\left(Y_{t+1} \geq \max \left(\gamma_{*} \lambda, Y_{t}(1-\varepsilon / 2)\right)\right) & \leq \operatorname{Pr}\left(Z \geq \max \left(\gamma_{*} \lambda, Y_{t}(1-\varepsilon / 2)\right)\right) \\
& \leq \operatorname{Pr}\left(Z \geq \mathbf{E}[Z]\left(1+\frac{\varepsilon}{2(1-\varepsilon)}\right)\right) \\
& \leq \exp \left(-\frac{\varepsilon^{2} \max \left(\gamma_{*} \lambda, Y_{t}\right)}{12(1-\varepsilon)}\right) \\
& \leq \exp \left(-\frac{\varepsilon^{2} \gamma_{*} \lambda}{12(1-\varepsilon)}\right) .
\end{aligned}
$$

The proof is completed by induction with respect to $t$ and a union bound.

Proof (of Theorem 8). We apply the level-based theorem with respect to a partitioning of the search space $\mathcal{X}=\{0,1\}^{n} \times \mathcal{M}$ into the following $n+2$ levels

$$
A_{j}:= \begin{cases}\left\{\left(0^{n}, \chi_{\text {low }}\right),\left(0^{n}, \chi_{\text {high }}\right)\right\} & \text { if } j=-1, \\ \left\{\left(x, \chi_{\text {low }}\right),\left(x, \chi_{\text {high }}\right) \mid \operatorname{Lo}(x)=0 \wedge x \neq 0^{n}\right\} & \text { if } j=0, \\ \left\{\left(x, \chi_{\text {low }}\right),\left(x, \chi_{\text {high }}\right) \mid \operatorname{Lo}(x)=j\right\} & \text { if } 1 \leq j \leq \ell-2, \\ \left\{\left(x, \chi_{\text {low }}\right),\left(y, \chi_{\text {high }}\right) \mid \operatorname{LO}(x)=\ell-1, \operatorname{LO}(y) \geq \ell-1\right\} & \text { if } j=\ell-1, \\ \left\{\left(x, \chi_{\text {low }}\right) \mid \operatorname{LO}(x)=j\right\} & \text { if } \ell \leq j \leq n .\end{cases}
$$

where $\ell \in[n]$ is the unique integer such that $\left(1-\frac{\chi_{\text {high }}}{n}\right)^{\ell}<\frac{85}{171} \leq\left(1-\frac{\chi_{\text {high }}}{n}\right)^{\ell-1}$. Note that as long as $m \leq \ln (171 / 85)(n-1) / \chi_{\text {high }}$, we have $\left(1-\frac{\chi_{\text {high }}}{n}\right)^{m} \geq$ $\left(e^{-\chi_{\text {high }}}\right)^{\frac{m}{n-1}} \geq \frac{85}{171}>\left(1-\frac{\chi_{\text {high }}}{n}\right)^{\ell}$, hence $\ell>m$.

We first estimate the expected runtime assuming that every population contains less than $\psi \lambda$ individuals in $A_{-1}$, and less than $\xi \lambda$ individuals in the set $B:=\left\{\left(y, \chi_{\text {high }}\right) \mid \operatorname{LO}(y) \geq \ell\right\}$, where $\psi:=123 / 250$ and $\xi:=1 / 5$. In the end, we will account for the generations where these assumptions do not hold. We begin by showing that condition (G2) of the level-based theorem hold for all levels.

Levels $0 \leq j \leq m$ : Assume that the population contains $\gamma \lambda$ individuals in levels $A_{\geq j}$ for any $\gamma \in\left(0, \gamma_{0}\right)$. An individual in $A_{\geq j}$ will be selected if the tournament contains at least one individual in $A_{\geq j}$, and no individuals in level $A_{-1}$. The probability of this event is $\beta(\gamma) \geq 2 \gamma\left(1-\gamma_{0} / 2-\psi\right)$. The mutated offspring of the selected individual will belong to levels $A_{\geq j}$ if none of the first $j \leq m$ bits are flipped, which occurs with probability at least $r(m)$. Hence, condition $(\mathrm{G} 2)$ is satisfied if there exists a $\gamma_{0} \in(0,1)$ and a constant $\delta>0$ such that for all $\gamma \in\left(0, \gamma_{0}\right]$, it holds $\beta(\gamma) r(m) \geq \gamma(1+\delta)$, i.e., it is sufficient to choose $m \in \mathbb{N}$ sufficiently small such that $r(m)=\left(1-\frac{\chi_{\text {high }}}{n}\right)^{m} \geq \frac{1+\delta}{2\left(1-\gamma_{0} / 2-\psi\right)}$. Note that such an $m=\Theta(n)$ exists, because $2\left(1-\gamma_{0} / 2-\psi\right)=127 / 125-\gamma_{0}>1+\delta$ when $\gamma_{0}$ and $\delta$ are sufficiently small.

Levels $m+1 \leq j<\ell$ : The probability of mutating an individual from $A_{\geq j}$ into $A_{\geq j}$, pessimistically assuming that the selected individual uses the high mutation rate $\chi_{\mathrm{high}}$, is at least $r(\ell-1)(1-p)+q(\ell-1) p>r(\ell-1)(1-p)+q(n) p>$ 
$(85 / 171)(1-p)+(2 / 3) p=1 / 2+1 / 180$. Hence, assuming that the current population has $\gamma \lambda$ individuals in $A_{\geq j}$ where $\gamma \in\left(0, \gamma_{0}\right)$, the probability of selecting one of these individuals and mutating them into $A_{\geq j}$ is at least $\beta(\gamma)(r(\ell-1)(1-$ $p)+q(\ell-1) p)>2 \gamma\left(1-\gamma_{0} / 2\right)(1 / 2+1 / 180)=\gamma\left(1-\gamma_{0} / 2\right)(1+1 / 90)>\gamma\left(1+\delta^{\prime}\right)$ for some $\delta^{\prime}>0$ given that $\gamma_{0}$ is a sufficiently small constant. Note that the lower bound on $\beta(\gamma)$ here does not depend on $\psi$, and nor on $\xi$ because in this setting the peak individuals have lower fitness than the individuals in $A_{j}$, and $B \subset A_{\geq j}$.

Levels $\ell \leq j \leq n$ : By the level-partitioning, any individual in these levels uses the low mutation rate $\chi_{\text {low }}$, and other individuals with at least $\ell$ leading 1-bits belong to the set $B$. Assume that the current population contains $\gamma \in\left(0, \gamma_{0}\right)$ individuals in levels $A_{\geq j}$. An individual in $A_{\geq j}$ can be produced by having a binary tournament with at least one individual from $A_{>j}$ and none of the at most $\xi \lambda$ individuals in $B$, not mutating any of the bits, and not changing the mutation rate. The probability of this event is at least $2 \gamma\left(1-\gamma_{0} / 2-\xi\right) q(n)(1-p) \geq$ $\gamma\left(4 / 5-\gamma_{0} / 2\right)(19 / 15)=\gamma\left(1+1 / 75-(19 / 30) \gamma_{0}\right)>\gamma\left(1+\delta^{\prime}\right)$ for some constant $\delta^{\prime}>0$, assuming that $\gamma_{0}$ is sufficiently small.

We now show that condition (G1) of the level-based theorem is satisfied for a parameter $z=\Omega(1 / n)$ in any level $j$. Assume that the current population contains at least $\gamma_{0} \lambda$ individuals in $A_{\geq j}$. Then, to create an individual in $A_{\geq j+1}$, it is sufficient to create a tournament of two individuals from $A_{\geq j}$, flip at most one bit, and either keep or switch the mutation rate. The probability of such an event is at least $\gamma_{0}^{2}\left(\chi_{\text {low }} / n\right)\left(1-\chi_{\text {high }} / n\right)^{n-1} p=\Omega(1 / n)$.

To complete the application of the level-based theorem, we note that since $\delta$ and $\gamma_{0}$ are constants, condition (G3) is satisfied when $\lambda \geq c \ln n$ for some constant $c$. Hence, under the assumptions on the number of individuals in level $A_{-1}$ and $B$ described above, the level-based theorem implies that the algorithm obtains the optimum in expected $t_{1}(n)=O\left(n \log (\lambda)+n^{2} / \lambda\right)$ generations. Furthermore, by Markov's inequality, the probability that the optimum has not been found within $2 t_{1}(n)$ generations is less than $1 / 2$.

To complete the proof, we justify the assumption that less than $\psi \lambda$ individuals belong to level $A_{-1}$, and less than $\xi \lambda$ individuals belong to $B$. We will show using Lemma 2 that starting with any population, these assumptions hold after an initial phase of $t_{0}(n)=O(\log (\lambda))$ generations. We call a phase good if the assumptions hold for the next $t_{1}(n)<e^{c \lambda}$ generations.

To apply Lemma 2 with respect to level $A_{-1}$, we note that the probability of obtaining an individual in $A_{-1}$ by mutating an individual in $A_{-1}$ is bounded from above by $q(n)(1-p)+r(n) p \leq(2 / 3) e^{\varepsilon}(1-p)+p / 3 \leq 65 / 99$. Furthermore, to mutate an individual from $\mathcal{X} \backslash A_{-1}$ into $A_{-1}$, it is necessary to flip at least one specific bit-position, i.e., with probability $O(1 / n)$. Therefore, by Lemma 2 with $\sigma=49 / 4950$ and $\rho=65 / 99$, it holds for all $t$ where $t_{0}(n)<t<e^{c n}$ and $t_{0}(n)=O(\log (\lambda))$ that $\operatorname{Pr}\left(\left|P_{t} \cap A_{-1}\right| \geq \psi \lambda\right)=e^{-\Omega(\lambda)}$ where $\psi:=123 / 250$.

Similarly, the probability of not destroying a $B$-individual with mutation is by definition of $\ell$ at most $\left(1-\frac{\chi_{\text {high }}}{n}\right)^{\ell}(1-p) \leq\left(\frac{85}{171}\right)\left(\frac{19}{20}\right)=\frac{17}{36}=: \rho$. To create a $B$ individual from $\mathcal{X} \backslash B$, it is in the best case necessary to change the mutation rate from $\chi_{\text {low }}$ to $\chi_{\text {high }}$ and not mutate the first $\ell$ bit-positions. The probability of this 
event is $\left(1-\frac{\chi_{\text {high }}}{n}\right)^{\ell} p \leq\left(\frac{85}{171}\right)\left(\frac{1}{20}\right)=\frac{17}{684}$. Therefore, by Lemma 2 with respect to $\sigma:=3 / 20$ and the above value of $\rho$, for every generation $t$ where $t_{0}(n)<t<e^{c \lambda}$ and $t_{0}(n)=O(\log (\lambda))$ it holds $\operatorname{Pr}\left(\left|P_{t} \cap B\right| \geq \xi \lambda\right)=e^{-\Omega(\lambda)}$, where $\xi:=1 / 5$.

To summarise, starting from any configuration of the population, a phase of length $t_{0}(n)+2 t_{1}(n)=O\left(n \log (\lambda)+n^{2} / \lambda\right)$ generations is good with probability $1-e^{-\Omega(\lambda)}$. If a phase is good, then the optimum will be found by the end of that phase with probability at least $1 / 2$. Hence, the expected number of phases required to find the optimum is $O(1)$, and the theorem follows, keeping in mind that each generation costs $\lambda$ function evaluations.

Below are results from 1000 experiments with the self-adaptive EA on the LEADINGONES function for $n=200, p=1 / 1000$ using $(\mu, \lambda)$-selection for $\mu=500, \lambda=4 \mu$, and mutation parameters $\mathcal{M}=\{2 / 5,2\}$. For each $j \in[n]$, the figure contains a box-plot describing the distribution of the fraction of the population choosing $\chi_{\text {low }}$ over all generations where the $(1 / 10)$-ranked individual in the population has $j$ leading one-bits.

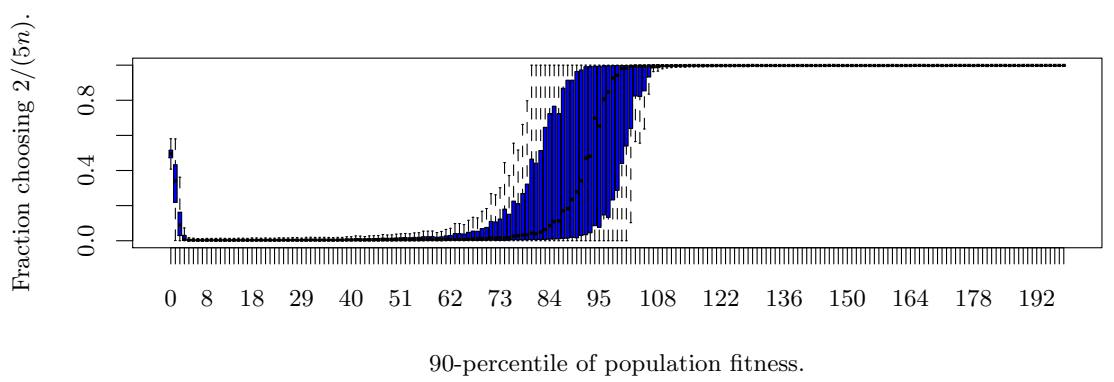

The initial population, including mutation rates, are sampled uniformly at random. Hence the (1/10)-ranked individual will have fitness close to 1 in the first generations. For $j \leq 5$, i. e. early in the run, approximately half of the population chooses the low mutation. However, the population quickly switches to the higher mutation $\chi_{\text {high }}$ until the $(1 / 10)$-ranked individual in the population reaches a value approximately $j \geq 60$ where the population switches to the lower mutation $\chi_{\text {low }}$. Almost all individuals choose $\chi_{\text {low }}$ for $j \geq 108$. These experimental results confirm that the population adapts the mutation rate according to the region of the fitness landscape currently searched.

\section{Conclusion}

This is the first rigorous runtime analysis of self-adaptation. We have demonstrated that self-adaptation with a sufficiently low strategy parameter can robustly control the mutation-rates of non-elitist EAs in discrete search spaces, and that this automated control can lead to exponential speedups compared to EAs that use fixed mutation rates, or uniform mixing of mutation rate. 
Acknowledgements This work received funding from the European Union Seventh Framework Programme (FP7/2007-2013) under grant agreement no. 618091 (SAGE).

\section{References}

1. T. Bäck. Self-adaptation in genetic algorithms. In Proc. of ECAL'92, pages 263-271, 1992.

2. S. Böttcher, B. Doerr, and F. Neumann. Optimal fixed and adaptive mutation rates for the leadingones problem. In Proc. of PPSN'10, pages 1-10, 2010.

3. D. Corus, D.-C. Dang, A. V. Eremeev, and P. K. Lehre. Level-based analysis of genetic algorithms and other search processes. In Proc. of PPSN'14, pages 912-921. Springer, 2014.

4. D.-C. Dang and P. K. Lehre. Refined upper bounds on the expected runtime of non-elitist populations from fitness-levels. In Proc. of GECCO'14, pages 1367-1374, 2014.

5. B. Doerr and C. Doerr. Optimal parameter choices through self-adjustment: Applying the 1/5-th rule in discrete settings. In Proc. of GECCO'15, pages 1335 1342,2015

6. B. Doerr, C. Doerr, and T. Kötzing. Solving problems with unknown solution length at (almost) no extra cost. In Proc of GECCO' 15, pages 831-838, New York, NY, USA, 2015. ACM.

7. A. E. Eiben, Z. Michalewicz, M. Schoenauer, and J. E. Smith. Parameter control in evolutionary algorithms. In Parameter Setting in Evolutionary Algorithms, pages 19-46. Springer, 2007.

8. N. Hansen and A. Ostermeier. Completely derandomized self-adaptation in evolution strategies. Evol. Comp., 9(2):159-195, 2001.

9. P. K. Lehre. Negative drift in populations. In Proc. of PPSN'10, pages 244-253, 2010.

10. P. K. Lehre and E. Özcan. A runtime analysis of simple hyper-heuristics: To mix or not to mix operators. In Proc. of FOGA'13, pages 97-104, 2013.

11. P. K. Lehre and X. Yao. On the impact of mutation-selection balance on the runtime of evolutionary algorithms. IEEE Trans. Evol. Comput., 16(2):225-241, 2012.

12. S. van Rijn, M. T. M. Emmerich, E. Reehuis, and T. Bäck. Optimizing highly constrained truck loadings using a self-adaptive genetic algorithm. In Proc. of CEC '15, pages 227-234, 2015.

13. J. Z. Xue, A. Kaznatcheev, A. Costopoulos, and F. Guichard. Fidelity drive: A mechanism for chaperone proteins to maintain stable mutation rates in prokaryotes over evolutionary time. J. Theor. Biol., 364:162-167, 2015. 


\section{Appendix A}

Lemma 3. For any $\delta \in(0,1)$ and $\chi>0$, if $n \geq(\chi+\delta)(\chi / \delta)$ then

$$
(1-\delta) e^{-\chi} \leq\left(1-\frac{\chi}{n}\right)^{n} \leq e^{-\chi}
$$

Proof. The upper bound follows immediately from the inequality $1+x \leq e^{x}$. For the lower bound, note first that $\ln (1-\delta)<-\delta$, hence

$$
\left(\frac{n}{\chi}-1\right)(\chi-\ln (1-\delta)) \geq n+\frac{n \delta}{\chi}-(\chi+\delta) \geq n .
$$

By making use of the fact that $(1-1 / x)^{x-1} \geq 1 / e$ and simplifying the exponent $n$ as above

$$
\left(1-\frac{\chi}{n}\right)^{n} \geq\left[\left(1-\frac{\chi}{n}\right)^{(n / \chi)-1}\right]^{\chi-\ln (1-\delta)} \geq(1-\delta) e^{-\chi}
$$

Lemma 4. Let $X \sim \operatorname{Bin}(\lambda, p)$ with $p \leq(k / \lambda)(1-\delta)$ for some $k \in[\lambda]$ and some $\delta \in[0,1)$, then

$$
\operatorname{Pr}(X>k) \leq \exp \left(-\frac{k^{2} \delta^{2}}{2 \lambda}\right) .
$$

Proof. Define $Y:=\lambda-X$, thus $Y \sim \operatorname{Bin}(\lambda, q:=1-p)$. Therefore, $q \geq \lambda-$ $(k / \lambda)(1-\delta)=(\lambda-k(1-\delta)) / \lambda$ and

$$
\mathbf{E}[Y] \geq \lambda-k(1-\delta) .
$$

Then by a Chernoff bound,

$$
\begin{aligned}
\operatorname{Pr}(X>k) & =\operatorname{Pr}(Y<\lambda-k) \\
& =\operatorname{Pr}\left(Y<(\lambda-k(1-\delta))\left(\frac{\lambda-k}{\lambda-k(1-\delta)}\right)\right) \\
& \leq \operatorname{Pr}\left(Y<\mathbf{E}[Y]\left(1-\frac{k \delta}{\lambda-k(1-\delta)}\right)\right) \\
& \leq \exp \left(-\left(\frac{k \delta}{\lambda-k(1-\delta)}\right)^{2} \frac{\mathbf{E}[Y]}{2}\right) \\
& \leq \exp \left(-\left(\frac{k \delta}{\lambda-k(1-\delta)}\right)^{2} \frac{\lambda-k(1-\delta)}{2}\right) \\
& =\exp \left(-\frac{k^{2} \delta^{2}}{2(\lambda-k(1-\delta))}\right)<\exp \left(-\frac{k^{2} \delta^{2}}{2 \lambda}\right) .
\end{aligned}
$$

